\title{
Studies on Correlation and Path Analysis for Yield and its Contributing Traits in Brinjal (Solanum melongena L.)
}

\author{
V. R. Gurve ${ }^{1 *}$, D. P. Waskar ${ }^{1}$, V. S. Khandare ${ }^{1}$, S. P. Mehtre ${ }^{2}$ and D. G. More ${ }^{2}$ \\ ${ }^{1}$ Department of Horticulture, ${ }^{2}$ AICRP on Soybean, Vasantrao Naik Marathwada Krishi \\ Vidyapeeth, Parbhani-431402, Maharashtra, India \\ *Corresponding author
}

\section{A B S T R A C T}

\begin{tabular}{l} 
Ke y w o r d s \\
$\begin{array}{l}\text { Brinjal, Genotypic } \\
\text { correlation, } \\
\text { Phenotypic } \\
\text { correlation, } \\
\text { Path analysis }\end{array}$ \\
\hline $\begin{array}{l}\text { Article Info } \\
\text { Accepted: } \\
\text { 04 September } 2020 \\
\text { Available Online: } \\
\text { 10 October } 2020\end{array}$ \\
\hline
\end{tabular}

\section{Introduction}

Brinjal (Solanum melongena L., $2 \mathrm{n}=24$ ), a member of the Solanaceae family, is the most common and popular vegetable crop of India. It grown in almost all parts of India yearround and is a major source of income for small and marginal farmers. It is being grown extensively in India, Bangladesh, Pakistan, China and Philippines. India is the second major producer of brinjal in the world after China (Ravali et al., 2017). Due to its highest production potential and availability of the produce to consumers, it is also called as poor man's vegetable (Kumar et al., 2014). India is a major producer of brinjal in the world and it is grown in area of 648 thousand hectares with estimated annual production of 12,303 thousand metric tonnes with a productivity of 18.98 metric tonnes per hectare. In Maharashtra, it is grown in an area of 35,859 hectare, with an annual production of 667,640 metric tonnes and productivity of 18.61 metric tonnes per hectare which is less than the national average (Anon., 2018-19).

An improvement in brinjal is normally achieved by selecting the genotypes with desirable character combination existing in the germplasm or by hybridization. 
Correlation and path co-efficient analysis are the important biometrical technique to determine the yield components. The characters that are positively correlated with yield are considerably important to plant breeder for selection purpose. Correlation provides a measure of genetic association between the characters and reveals the traits that might be useful as an index of selection. A study of association of these characters helps in selection of genotypes and also suggests the advantage of a selection scheme for more than one character at a time, which could be explained that improvement of one character results in improvement of all positively related characters. In the present study, the simple correlation coefficients between yield and its components and their inter correlations among the components were estimated. Although the correlation coefficient indicates the nature of association among the different traits, path analysis splits the correlation co-efficient into measure of direct and indirect effects thus providing understanding of the direct and indirect contribution of each character towards yield. Hence, the present study was under taken to unravel the correlation and path co-efficient of yield and yield attributing traits in brinjal.

\section{Materials and Methods}

The present investigation was carried out at Horticulture Research Scheme (Vegetable), Department of Horticulture, VNMKV, Parbhani, during the kharif season, 2017-18. The experiment was laid out in a Randomized Block Design with two replications. The experimental material comprised of twenty genotypes collected from IIVR, Varanasi and VNMKV, Parbhani. The 42 days old healthy seedlings of each genotype were transplanted with a spacing of $75 \mathrm{~cm} x \quad 60 \mathrm{~cm}$. The recommended package of practices was adopted for raising the crop. Observation were recorded in five randomly selected plants for fourteen characters viz. plant height (cm), number of branches per plant, plant spread $(\mathrm{cm})$, days to $50 \%$ flowering, fruit setting (\%), number of fruit per plant, days to last harvest, fruit length $(\mathrm{cm})$, fruit diameter $(\mathrm{cm})$, fruit pedicel length $(\mathrm{cm})$, ascorbic acid (mg/100g), total phenolic content ( $\mathrm{mg} / 100 \mathrm{~g}$ ), flavonoid content $(\mathrm{mg} / 100 \mathrm{~g})$ and fruit yield per plant. Analysis of covariance for all combinations were done and used for estimation of correlations. The formula as suggested by Johnson et al., (1955) was used for calculating simple correlation coefficient. Path analysis was done as per the procedure outlined by Dewey and Lu (1959).

\section{Results and Discussion}

In general, it was revealed that genotypic correlation coefficients were higher than phenotypic correlation coefficients. This could be interpreted on the basis that there was a strong inherent genotypic relationship between the characters studied, but their phenotypic expression was impeded by the influence of environmental factors.Plant height were exhibited positive significant correlation with number of branches per plant (0.1756 P, $0.2414 \mathrm{G})$, plant spread (N-S) $(0.3567 \mathrm{P}, 0.6487 \mathrm{G})$ and $(\mathrm{E}-\mathrm{W})(0.4954 \mathrm{P}$, $0.6720 \mathrm{G})$, number of fruit per plant $(0.2495$ $\mathrm{P}, 0.2470 \mathrm{G})$, fruit length $(0.4871 \mathrm{P}, 0.6231$ $\mathrm{G})$, fruit pedicel length $(0.5174 \mathrm{P}, 0.7608 \mathrm{G})$, ascorbic acid $(0.2236 \mathrm{P}, 0.3226 \mathrm{G})$ and fruit yield per plant $(0.3115 \mathrm{P}, 0.2837 \mathrm{G})$.Ascorbic acid $(0.3226 \mathrm{G})$ has recorded positive and significant association with plant height at genotypic level. In present experiment, it was observed that as the plant height increased, there was corresponding increase in number of branches per plant, plant spread, fruit length, fruit pedicel length, ascorbic acid and number of fruits per plant. The association recorded significant improvement in yield. Similar results were obtained in brinjal by (Saleem et al., 2013). 
Number of primary branches per plant were recorded positive, significant correlation with plant spread $(\mathrm{N}-\mathrm{S})(0.2298 \mathrm{P}, 0.6638 \mathrm{G})$ and $(\mathrm{E}-\mathrm{W})(0.1431 \mathrm{P}, 0.2928 \mathrm{G})$, number of fruit per plant $(0.3106 \mathrm{P}, 0.3716 \mathrm{G})$ and fruit yield per plant $(0.4259 \mathrm{P}, 0.5364 \mathrm{G})$. Negative and significant correlation was observed with days to $50 \%$ flowering $(-0.2767 \mathrm{P},-0.2568 \mathrm{G})$, Flavonoid content $(-0.1846 \mathrm{G})$ were exhibited significant and negative association with number of branches per plant at genotypic level.

Days to 50 per cent flowering showed significant negative correlation with fruit diameter $(-0.2888 \mathrm{P},-0.3093 \mathrm{G})$, fruit pedicel length $(-0.3207 \mathrm{P},-0.3328 \mathrm{G})$ and fruit yield per plant $(-0.3496 \mathrm{P},-0.4629 \mathrm{G})$ and showed significant positive correlation with days to last harvest $(0.1421 \mathrm{P}, 0.2967 \mathrm{G})$. Number of branches per plant shows significant and positive association with fruit yield per plant and negative significant correlation was also noticed with days to $50 \%$ flowering. Similar reports were also noticed by Umar Musa Tanko Momoh and Jimoh Yusuf (2015) and Madhavi et al., (2015), Sawadogo et al., (2016) in brinjal.

Number of fruit per plant showed positive significant correlation with fruit yield per plant $(0.5352 \mathrm{P}, 0.5738 \mathrm{G})$. Negative and significant correlation was noticed with days to last harvest $(-0.3031 \mathrm{P},-0.3660 \mathrm{G})$, and fruit diameter $(-0.3833 \mathrm{P},-0.4416 \mathrm{G})$. Length of fruit were recorded positive, significant correlation with fruit pedicel length $(0.6182$ $\mathrm{P}, 0.6633 \mathrm{G})$, and fruit yield per plant $(0.3171$ $\mathrm{P}, 0.3259 \mathrm{G})$.

Total phenolic content $(-0.5710 \mathrm{G})$ and flavonoid content $(-0.2222 \mathrm{G})$ has recorded negative and significant association was noticed with length of fruit at genotypic level. Diameter of fruit was recorded positive, significant correlation withascorbic acid $(0.2707 \mathrm{P}, 0.2804 \mathrm{G})$ and fruit yield per plant
(0.1633 P, $0.1471 \mathrm{G})$. Ascorbic acid content showed positive significant correlation with flavonoid content $(0.2461 \mathrm{P}, 0.2484 \mathrm{G})$. Fruit yield per plant was recorded positive significant correlation with characters plant height ( $0.3115 \mathrm{P}, 0.2837 \mathrm{G})$, number of branches per plant $(0.4259 \mathrm{P}, 0.5364 \mathrm{G})$, plant spread (N-S) (0.5880 P, $0.8043 \mathrm{G})$ and (E-W) $(0.5338 \mathrm{P}, 0.5782 \mathrm{G})$ and fruit length $(0.3171$ $\mathrm{P}, 0.3259 \mathrm{G})$. Days to $50 \%$ flowering $(-0.3496$ $\mathrm{P},-0.4629 \mathrm{G})$ and days to last harvest(-0.2347 $\mathrm{P},-0.3346 \mathrm{G})$ has recorded negative and significant association with fruit yield per plant at phenotypic and genotypic level. Fruit yield per plant had exhibited highly significant and positive association with fruit circumference and fruit weight were recorded by Gupta et al., (2015). The number of branches per plant and number of fruit per plant were significantly and positively associated with fruit yield both at phenotypic as well as genotypic level. Similar result was recorded by Pandey et al., (2016).

Fruit yield per plant exhibited high significant positive association with plant height, number of branches per plant, plant spread, number of fruit per plant, fruit length, fruit diameter, fruit pedicel length and ascorbic acid. Number of fruits per plant, fruit length and diameter of fruit indicating the importance of these traits in selection for yield.

Direct selection based on these traits would result in simultaneous improvement of aforesaid traits and yield in brinjal. Similar results were reported in brinjal for different components viz., association of fruit yield per plant with the number of branches per plant Samadia (2007), fruit length and fruit diameter (Shinde et al., 2009), plant height and number of fruit per plant (Saleem et al., 2013), association of fruit yield with number of branches (Patel et al., 2015), number of fruit per plant and fruit weight, Reshmika (2015), and number of fruit per plant and fruit diameter (Sujin et al., 2017) (Table 1-4). 
Table.1 Genotypical correlation coefficient for different fruit yield contributing characters in brinjal

\begin{tabular}{|c|c|c|c|c|c|c|c|c|c|c|c|c|c|c|c|}
\hline Characters & $\begin{array}{c}\text { Plant } \\
\text { height } \\
(\mathrm{cm})\end{array}$ & $\begin{array}{c}\text { Number of } \\
\text { branches } \\
\text { per plant }\end{array}$ & $\begin{array}{c}\text { Plant sp } \\
\text { NS }\end{array}$ & $\mathbf{a d}(\mathbf{c m})$ & $\begin{array}{c}\text { Days to } \\
50 \% \\
\text { flowering }\end{array}$ & $\begin{array}{c}\text { Fruit } \\
\text { setting } \\
\%\end{array}$ & $\begin{array}{c}\text { Number of } \\
\text { fruit per } \\
\text { plant }\end{array}$ & $\begin{array}{c}\text { Days to } \\
\text { last } \\
\text { harvest }\end{array}$ & $\begin{array}{l}\text { Fruit } \\
\text { length } \\
\text { (cm) }\end{array}$ & $\begin{array}{c}\text { Fruit } \\
\text { diameter } \\
(\mathbf{c m})\end{array}$ & $\begin{array}{c}\text { Fruit } \\
\text { pedicel } \\
\text { length } \\
(\mathrm{cm})\end{array}$ & $\begin{array}{l}\text { Ascorbic } \\
\text { acid (mg) }\end{array}$ & $\begin{array}{l}\text { Total } \\
\text { phenolic } \\
\text { content }\end{array}$ & $\begin{array}{c}\text { Flavonoid } \\
\text { content }\end{array}$ & $\begin{array}{c}\text { Fruit yield } \\
\text { per plant } \\
\text { (g) }\end{array}$ \\
\hline Plant height (cm) & $\begin{array}{c}1.000 \\
0\end{array}$ & $0.2414 *$ & $\begin{array}{c}0.6487 * \\
*\end{array}$ & $\begin{array}{l}0.6720 \\
* *\end{array}$ & -0.1859 & $\begin{array}{c}- \\
0.4784 \\
* *\end{array}$ & $0.2495^{*}$ & -0.0144 & $\begin{array}{c}0.6231^{*} \\
*\end{array}$ & $-0.3910 * *$ & $0.7608^{* *}$ & $0.3226^{* *}$ & $-0.2257^{*}$ & -.0 .0612 & $0.2837^{*}$ \\
\hline $\begin{array}{l}\text { Number of branches } \\
\text { per plant }\end{array}$ & & 1.0000 & $\begin{array}{c}0.6638^{*} \\
*\end{array}$ & $\begin{array}{c}0.2928 \\
*\end{array}$ & $-0.2568 *$ & $\begin{array}{c}- \\
0.0797\end{array}$ & $0.3716^{* *}$ & -0.0826 & 0.0134 & 0.0542 & 0.1669 & -0.0086 & 0.0089 & -0.1846 & $0.5364 * *$ \\
\hline \multirow[t]{2}{*}{$\begin{array}{l}\text { Plant spread }(\mathrm{cm}) \\
\text { NS }\end{array}$} & & & 1.0000 & $\begin{array}{c}1.0292 \\
* *\end{array}$ & -0.1607 & $\begin{array}{c}- \\
0.1299\end{array}$ & $0.4065^{* *}$ & $0.2411^{*}$ & 0.0458 & -0.1454 & -0.1859 & 0.0022 & $0.2785^{*}$ & -0.0337 & $0.8043 * *$ \\
\hline & & & & 1.0000 & -0.1333 & $\begin{array}{l}- \\
0.2355 \\
*\end{array}$ & $0.2441^{* *}$ & 0.1670 & 0.1689 & -0.1395 & 0.0856 & 0.1840 & $0.2144 *$ & $0.2147^{*}$ & $0.5782 * *$ \\
\hline Days to $50 \%$ flowering & & & & & 1.0000 & 0.0914 & $-0.2028 *$ & $0.2967 * *$ & 0.0414 & $-0.3093 * *$ & $-0.3328 * *$ & 0.0448 & -0.1093 & 0.0413 & $-0.4629 * *$ \\
\hline Fruit setting \% & & & & & & 1.0000 & $-0.2469 *$ & -0.0235 & -0.0303 & $0.3289 * *$ & -0.0687 & 0.1178 & 0.1811 & $0.3042 * *$ & 0.0269 \\
\hline $\begin{array}{l}\text { Number of fruit per } \\
\text { plant }\end{array}$ & & & & & & & 1.0000 & $-0.3660 * *$ & 0.1246 & $-0.4416^{* *}$ & 0.1133 & -0.0547 & $0.2462 *$ & -0.0195 & $0.5738 * *$ \\
\hline Days to last harvest & & & & & & & & 1.0000 & $-0.2947 *$ & -0.0305 & $-0.4988 * *$ & -0.1468 & 0.1101 & 0.1968 & $-0.3346 * *$ \\
\hline Fruit length (cm) & & & & & & & & & 1.0000 & $-0.4460 * *$ & $0.6633^{* *}$ & -0.0046 & $-0.5710 * *$ & $-0.2222 * *$ & $0.3259 * *$ \\
\hline Fruit diameter $(\mathrm{cm})$ & & & & & & & & & & 1.0000 & $-0.4283^{* *}$ & $0.2804 *$ & 0.1226 & 0.0105 & 0.1471 \\
\hline $\begin{array}{l}\text { Fruit pedicel length } \\
\text { (cm) }\end{array}$ & & & & & & & & & & & 1.0000 & -0.0435 & $-0.4144 * *$ & $-0.2784^{*}$ & 0.1361 \\
\hline Ascorbic acid (mg) & & & & & & & & & & & & 1.0000 & 0.1558 & $0.2484 *$ & 0.1072 \\
\hline Total phenolic content & & & & & & & & & & & & & 1.0000 & $0.5987 * *$ & 0.0652 \\
\hline Flavonoid content & & & & & & & & & & & & & & 1.0000 & -0.1199 \\
\hline $\begin{array}{l}\text { Fruit yield per plant } \\
\text { (g) }\end{array}$ & & & & & & & & & & & & & & & 1.0000 \\
\hline
\end{tabular}

* Significant at 5 per cent level, ** Significant at 1 per cent level 
Table.2 Phenotypical correlation coefficient for different fruit yield contributing characters in brinjal

\begin{tabular}{|c|c|c|c|c|c|c|c|c|c|c|c|c|c|c|c|}
\hline Characters & $\begin{array}{l}\text { Plant } \\
\text { height } \\
(\mathbf{c m})\end{array}$ & $\begin{array}{l}\begin{array}{c}\text { Number } \\
\text { of } \\
\text { branches }\end{array} \\
\text { per plant }\end{array}$ & Plant sp & $\operatorname{ad}(\mathbf{c m})$ & $\begin{array}{c}\text { Days to } \\
50 \% \\
\text { flowering }\end{array}$ & $\begin{array}{c}\text { Fruit } \\
\text { setting } \\
\%\end{array}$ & $\begin{array}{c}\text { Number } \\
\text { of fruit } \\
\text { per } \\
\text { plant }\end{array}$ & $\begin{array}{c}\text { Days to } \\
\text { last } \\
\text { harvest }\end{array}$ & $\begin{array}{l}\text { Fruit } \\
\text { length } \\
\text { (cm) }\end{array}$ & $\begin{array}{l}\text { Fruit } \\
\text { diameter } \\
\text { (cm) }\end{array}$ & $\begin{array}{l}\text { Fruit } \\
\text { pedicel } \\
\text { length } \\
(\mathrm{cm})\end{array}$ & $\begin{array}{l}\text { Ascorbi } \\
\text { c acid } \\
\text { (mg) }\end{array}$ & $\begin{array}{l}\text { Total } \\
\text { phenolic } \\
\text { content }\end{array}$ & $\begin{array}{l}\text { Flavonoid } \\
\text { content }\end{array}$ & $\begin{array}{l}\text { Fruit yield } \\
\text { per plant } \\
\text { (g) }\end{array}$ \\
\hline Plant height (cm) & 1.0000 & 0.1756 & $0.3567 * *$ & $0.4954 * *$ & -0.1570 & -0.1943 & $0.2470^{*}$ & -0.0452 & $0.4871 * *$ & $\stackrel{-}{-}$ & $0.5174 * *$ & $0.2236^{*}$ & -0.1720 & -0.0466 & $0.3115^{* *}$ \\
\hline $\begin{array}{l}\text { Number of branches } \\
\text { per plant }\end{array}$ & & 1.0000 & $0.2298 *$ & 0.1431 & $-0.2767 *$ & 0.0301 & $0.3106^{* *}$ & -0.0311 & -0.0063 & 0.0419 & 0.1700 & -0.0054 & 0.0082 & -0.1716 & $0.4259 * *$ \\
\hline Plant spread (cm) & & & 1.0000 & $0.7137 * *$ & -0.0459 & $-0.2187^{*}$ & $0.3555 * *$ & 0.1673 & 0.0729 & -0.0041 & 0.0419 & 0.0106 & 0.1544 & -0.0187 & $0.5880 * *$ \\
\hline $\begin{array}{l}\text { NS } \\
\text { EW }\end{array}$ & & & & 1.0000 & -0.0508 & $-0.3345 * *$ & $0.3208^{* *}$ & 0.1847 & 0.1782 & -0.0465 & 0.1750 & 0.1609 & 0.1734 & 0.1737 & $0.5338^{* *}$ \\
\hline Days to $50 \%$ flowering & & & & & 1.0000 & 0.0501 & -0.1336 & 0.1421 & 0.0204 & $-0.2888^{*}$ & $\stackrel{-}{-}$ & 0.0642 & -0.0985 & 0.0372 & $-0.3496^{* *}$ \\
\hline Fruit setting \% & & & & & & 1.0000 & $-0.2327 *$ & 0.0091 & -0.0318 & $0.2313^{*}$ & -0.0403 & 0.0464 & 0.1306 & $0.2194 *$ & -0.0195 \\
\hline $\begin{array}{l}\text { Number of fruit per } \\
\text { plant }\end{array}$ & & & & & & & 1.0000 & $-0.3031 * *$ & 0.1298 & $-0.3833^{* *}$ & 0.1266 & -0.0399 & $0.2332 *$ & -0.0185 & $0.5352 * *$ \\
\hline Days to last harvest & & & & & & & & 1.0000 & $-0.2406^{*}$ & 0.0158 & $-0.3087 * *$ & -0.1477 & 0.0989 & 0.1768 & $-0.2347 *$ \\
\hline Fruit length (cm) & & & & & & & & & 1.0000 & $-0.3973 * *$ & $0.6182 * *$ & 0.0003 & $-0.5605 * *$ & $-0.2181 *$ & $0.3171 * *$ \\
\hline Fruit diameter (cm) & & & & & & & & & & 1.0000 & $-0.2499 *$ & $0.2707^{*}$ & 0.1192 & 0.0102 & 0.1633 \\
\hline $\begin{array}{l}\text { Fruit pedicel length } \\
\text { (cm) }\end{array}$ & & & & & & & & & & & 1.0000 & -0.0228 & $-0.3528 * *$ & $-0.2370^{*}$ & 0.1377 \\
\hline Ascorbic acid (mg) & & & & & & & & & & & & 1.0000 & 0.1544 & $0.2461 *$ & 0.1020 \\
\hline Total phenolic content & & & & & & & & & & & & & 1.0000 & $0.5987 * *$ & 0.0579 \\
\hline Flavonoid content & & & & & & & & & & & & & & 1.0000 & -0.1065 \\
\hline Fruit yield per plant (g) & & & & & & & & & & & & & & & 1.0000 \\
\hline
\end{tabular}

* Significant at 5 per cent level, ** Significant at 1 per cent level 
Table.3 Genotypic $(\mathrm{G})$ path coefficient analysis indicating direct and indirect effects of components characters on fruit yield per plant in brinjal genotypes

\begin{tabular}{|c|c|c|c|c|c|c|c|c|c|c|c|c|c|c|c|}
\hline \multirow[t]{2}{*}{ Characters } & \multirow{2}{*}{$\begin{array}{c}\text { Plant } \\
\text { Height } \\
(\text { cm) }\end{array}$} & \multirow{2}{*}{$\begin{array}{c}\begin{array}{c}\text { Number } \\
\text { of } \\
\text { branches }\end{array} \\
\text { per plant }\end{array}$} & \multicolumn{2}{|c|}{$\begin{array}{l}\text { Plant spread } \\
\text { (cm) }\end{array}$} & \multirow{2}{*}{$\begin{array}{c}\text { Days to } \\
50 \% \\
\text { flowering }\end{array}$} & \multirow{2}{*}{$\begin{array}{c}\text { Fruit } \\
\text { setting } \\
\%\end{array}$} & \multirow{2}{*}{$\begin{array}{c}\text { Number } \\
\text { of fruit } \\
\text { per plant }\end{array}$} & \multirow{2}{*}{$\begin{array}{l}\text { Days to } \\
\text { last } \\
\text { harvest }\end{array}$} & \multirow{2}{*}{$\begin{array}{c}\text { Fruit } \\
\text { length } \\
(\mathrm{cm})\end{array}$} & \multirow{2}{*}{$\begin{array}{c}\text { Fruit } \\
\text { diameter } \\
(\mathbf{c m})\end{array}$} & \multirow{2}{*}{$\begin{array}{c}\text { Fruit } \\
\text { pedicel } \\
\text { length } \\
(\mathrm{cm})\end{array}$} & \multirow[t]{2}{*}{$\begin{array}{l}\text { Ascorbic } \\
\text { acid (mg) }\end{array}$} & \multirow{2}{*}{$\begin{array}{l}\text { Total } \\
\text { phenolic } \\
\text { content }\end{array}$} & \multirow[t]{2}{*}{$\begin{array}{l}\text { Flavonoid } \\
\text { content }\end{array}$} & \multirow{2}{*}{$\begin{array}{c}\text { Fruit } \\
\text { yield per } \\
\text { plant }(\mathrm{g})\end{array}$} \\
\hline & & & NS & EW & & & & & & & & & & & \\
\hline Plant height (cm) & -0.2734 & -0.0660 & -0.1773 & -0.1837 & 0.0508 & 0.1308 & -0.0682 & 0.0039 & -0.1704 & 0.1069 & -0.2080 & -0.0882 & 0.0617 & 0.0167 & 0.2837 \\
\hline $\begin{array}{c}\text { Number of branches } \\
\text { per plant }\end{array}$ & 0.0202 & 0.0836 & 0.0555 & 0.0245 & -0.0215 & -0.0067 & 0.0311 & -0.0069 & 0.0011 & 0.0045 & 0.0140 & -0.0007 & 0.0007 & -0.0154 & 0.5364 \\
\hline \multirow{2}{*}{$\begin{array}{c}\text { Plant spread (cm) } \\
\text { NS }\end{array}$} & 0.1477 & 0.1512 & 0.2277 & 0.2344 & -0.0366 & -0.0296 & 0.0926 & 0.0549 & 0.0104 & -0.0331 & -0.0423 & 0.0005 & 0.0634 & -0.0077 & 0.1832 \\
\hline & -0.9570 & -0.4169 & -1.4658 & -1.4242 & 0.1898 & 0.3355 & -0.4046 & -0.2379 & -0.2406 & 0.1986 & -0.1219 & -0.2621 & -0.3053 & -0.3058 & -0.8235 \\
\hline EW & & & & & & & & & & & & & & & \\
\hline Days to $50 \%$ flowering & -0.0149 & -0.0206 & -0.0129 & -0.0107 & 0.0803 & 0.0073 & -0.0163 & 0.0238 & 0.0033 & -0.0249 & -0.0267 & 0.0036 & -0.0088 & 0.0033 & -0.0372 \\
\hline Fruit setting \% & 2.0778 & 0.3461 & 0.5644 & 1.0230 & -0.3968 & -4.3430 & 1.0721 & 0.1022 & 0.1314 & -1.4283 & 0.2984 & -0.5115 & -0.7865 & -1.3212 & 0.0269 \\
\hline $\begin{array}{c}\text { Number of fruit per } \\
\text { plant }\end{array}$ & 0.2078 & 0.3095 & 0.3386 & 0.2366 & -0.1689 & -0.2056 & 0.8330 & -0.3049 & 0.1041 & -0.3678 & 0.0943 & 0.0456 & 0.2051 & -0.0163 & 0.5352 \\
\hline Days to last harvest & 0.1049 & 0.6001 & -1.7504 & -1.2129 & -2.1545 & 0.1709 & 2.6577 & -7.2615 & 2.1400 & 0.2215 & 3.6221 & 1.0662 & -0.7996 & -1.4289 & -0.3346 \\
\hline Fruit length (cm) & -0.4883 & -0.0105 & -0.0359 & -0.1324 & -0.0324 & 0.0237 & -0.0979 & 0.2309 & -0.7836 & 0.3494 & -0.5197 & 0.0036 & 0.4474 & 0.1741 & 0.3259 \\
\hline Fruit diameter $(\mathrm{cm})$ & 0.1810 & -0.0251 & 0.0673 & 0.0646 & 0.1432 & -0.1523 & 0.2045 & 0.0141 & 0.2065 & -0.4630 & 0.1983 & -0.1298 & -0.0568 & -0.0048 & 0.1471 \\
\hline $\begin{array}{c}\text { Fruit pedicel length } \\
(\mathrm{cm})\end{array}$ & 0.1197 & 0.0263 & -0.0293 & 0.0135 & -0.0524 & -0.0108 & 0.0178 & -0.0785 & 0.1044 & -0.0674 & 0.1574 & -0.0069 & -0.0652 & -0.0438 & 0.1361 \\
\hline Ascorbic acid (mg) & 0.0883 & -0.0023 & 0.0006 & 0.0504 & 0.0123 & 0.0322 & -0.0150 & -0.0402 & -0.0013 & 0.0767 & -0.0119 & 0.2737 & 0.0426 & 0.0680 & 0.1072 \\
\hline Total phenolic content & 0.0664 & -0.0026 & -0.0820 & -0.0631 & 0.0322 & -0.0533 & -0.0725 & -0.0324 & 0.1681 & -0.0361 & 0.1220 & -0.0459 & -0.2944 & -0.1763 & 0.0652 \\
\hline Flavonoid content & 0.0297 & 0.0897 & 0.0164 & -0.1043 & -0.0201 & -0.1478 & 0.0095 & -0.0956 & 0.1079 & -0.0051 & 0.1352 & -0.1207 & -0.2908 & -0.4857 & -0.1199 \\
\hline
\end{tabular}

Residual effect $=0.2227$ 
Table.4 Phenotypic (P) path coefficient analysis indicating direct and indirect effects of components characters on fruit yield per plant in brinjal genotypes

\begin{tabular}{|c|c|c|c|c|c|c|c|c|c|c|c|c|c|c|c|}
\hline Characters & $\begin{array}{c}\text { Plant } \\
\text { Height } \\
(\mathrm{cm})\end{array}$ & $\begin{array}{l}\text { Number of } \\
\text { branches } \\
\text { per plant }\end{array}$ & Plant sF & $\operatorname{cad}(\mathrm{cm})$ & $\begin{array}{c}\text { Days to } \\
50 \% \\
\text { flowering }\end{array}$ & $\begin{array}{c}\text { Fruit } \\
\text { setting \% }\end{array}$ & $\begin{array}{c}\text { Number of } \\
\text { fruit per plant }\end{array}$ & $\begin{array}{c}\text { Days to last } \\
\text { harvest }\end{array}$ & $\begin{array}{l}\text { Fruit } \\
\text { length } \\
\text { (cm) }\end{array}$ & $\begin{array}{c}\text { Fruit } \\
\text { diameter } \\
(\mathrm{cm})\end{array}$ & $\begin{array}{c}\text { Fruit } \\
\text { pedicel } \\
\text { length }(\mathbf{c m})\end{array}$ & $\begin{array}{l}\text { Ascorbic } \\
\text { acid (mg) }\end{array}$ & $\begin{array}{l}\text { Total } \\
\text { phenolic } \\
\text { content }\end{array}$ & $\begin{array}{c}\text { Flavonoid } \\
\text { content }\end{array}$ & $\begin{array}{c}\text { Fruit yield } \\
\text { per plant } \\
\text { (g) }\end{array}$ \\
\hline Plant height (cm) & -0.1639 & -0.0288 & -0.0585 & -0.0812 & 0.0257 & 0.0318 & -0.0405 & 0.0074 & -0.0799 & 0.0493 & -0.0848 & -0.0367 & 0.0282 & 0.0076 & 0.3115 \\
\hline $\begin{array}{c}\text { Number of branches } \\
\text { per plant }\end{array}$ & 0.0242 & 0.1379 & 0.0317 & 0.0197 & -0.0382 & 0.0042 & 0.0428 & -0.0043 & -0.0009 & 0.0058 & 0.0235 & -0.0007 & 0.0011 & -0.0237 & 0.4259 \\
\hline \multirow{2}{*}{$\begin{array}{c}\text { Plant spread (cm) } \\
\text { NS }\end{array}$} & 0.0402 & 0.0259 & 0.1126 & 0.0803 & -0.0052 & -0.0246 & 0.0400 & 0.0188 & 0.0082 & -0.0005 & 0.0047 & 0.0012 & 0.0174 & -0.0021 & 0.5880 \\
\hline & -0.2686 & -0.0776 & -0.3869 & -0.5421 & 0.0275 & 0.1813 & -0.1739 & -0.1001 & -0.0966 & 0.0252 & -0.0949 & -0.0872 & -0.0940 & -0.0942 & 0.5338 \\
\hline Days to $50 \%$ flowering & -0.0077 & -0.0136 & -0.0023 & -0.0025 & 0.0493 & 0.0025 & -0.0066 & 0.0070 & 0.0010 & -0.0142 & -0.0158 & 0.0032 & -0.0049 & 0.0018 & -0.3496 \\
\hline Fruit setting \% & 0.4566 & -0.0708 & 0.5141 & 0.7861 & -0.1179 & -2.3503 & 0.5469 & -0.0215 & 0.0748 & -0.5437 & 0.0947 & -0.1092 & -0.3070 & -0.5156 & -0.0195 \\
\hline $\begin{array}{c}\text { Number of fruit per } \\
\text { plant }\end{array}$ & 0.1731 & 0.2177 & 0.2492 & 0.2248 & -0.0937 & -0.1631 & 0.7009 & -0.2159 & 0.0910 & -0.2686 & 0.0887 & -0.0279 & 0.1634 & -0.0130 & 0.5738 \\
\hline Days to last harvest & 0.1702 & 0.1171 & -0.6309 & -0.6963 & -0.5359 & -0.0345 & 1.1617 & -3.7703 & 0.9071 & -0.0595 & 1.1638 & 0.5567 & -0.3730 & -0.6665 & -0.2347 \\
\hline Fruit length $(\mathrm{cm})$ & -0.0969 & 0.0013 & -0.0145 & -0.0355 & -0.0041 & 0.0063 & -0.0258 & 0.0479 & -0.1990 & 0.0791 & -0.1230 & 0.0001 & 0.1115 & 0.0434 & 0.3171 \\
\hline Fruit diameter $(\mathrm{cm})$ & 0.0855 & -0.0119 & 0.0012 & 0.0132 & 0.0820 & -0.0657 & 0.1088 & -0.0045 & 0.1128 & -0.2840 & 0.0710 & -0.0769 & -0.0339 & -0.0029 & 0.1633 \\
\hline $\begin{array}{l}\text { Fruit pedicel length } \\
\text { (cm) }\end{array}$ & 0.0117 & 0.0038 & 0.0009 & 0.0039 & -0.0072 & -0.0009 & 0.0029 & -0.0070 & 0.0140 & -0.0056 & 0.0226 & -0.0005 & -0.0080 & -0.0054 & 0.1377 \\
\hline Ascorbic acid (mg) & 0.0019 & 0.0000 & 0.0001 & 0.0013 & 0.0005 & 0.0004 & -0.0003 & -0.0012 & 0.0000 & 0.0023 & -0.0002 & 0.0084 & 0.0013 & 0.0021 & 0.1020 \\
\hline Total phenolic content & -0.0180 & 0.0009 & 0.0161 & 0.0181 & -0.0103 & 0.0136 & 0.0244 & 0.0103 & -0.0586 & 0.0125 & -0.0369 & 0.0162 & 0.1045 & 0.0626 & 0.0579 \\
\hline Flavonoid content & 0.0138 & 0.0507 & 0.0055 & -0.0513 & -0.0110 & -0.0648 & 0.0055 & -0.0522 & 0.0644 & -0.0030 & 0.0700 & -0.0727 & -0.1768 & -0.2953 & -0.1065 \\
\hline
\end{tabular}

Residual effect $=0.2725$ 
The overall perusal of correlation analysis results in brinjal indicated that traits like plant height, number of branches per plant, plant spread, number of fruit per plant, days to 50 $\%$ flowering, fruit length and fruit diameter had significant association with fruit yield per plant which indicated that the adequate knowledge of interrelationship between fruit yield per plant and its components themselves is useful for selection and simultaneous improvement in these characters. Similar results were reported in brinjal by Umar Musa Tanko Momoh and Jimoh Yusuf (2015) Sawadogo et al., (2016) and Shivkumar et al., (2016).

The estimation of correlation indicates only the extent and nature of association between yield and its components but does not show the direct and indirect effects of different yield attributes on yield per se. Fruit yield is dependent on several characters which are mutually associated; these will in turn impair the true association existing between a component and fruit yield. A change in any one component is likely to disturb the whole network of cause and effect. Thus, each component has two paths of action viz., the direct influence on fruit yield, indirect effect through components which are not revealed from the correlation studies (Lenka and Mishra 1973).

Number of branches had a positive direct effect (0.0836) on fruit yield per plant. It showed a maximum positive indirect effect through characters viz., plant spread(N-S) (0.0555), number of fruit per plant (0.0311) and plant spread (E-W) (0.0245) followed by plant height (0.0202). Positive direct effect (0.0803) on fruit yield was exerted by days to 50 per cent flowering and it had maximum positive indirect effect on fruit yield through days to last harvest $(0.0238)$ followed by fruit setting percent (0.0073), and ascorbic acid (0.0036). Number of fruits per plant had a positive direct effect $(0.8330)$ on fruit yield per plant. While, it showed maximum negative indirect effect through fruit diameter $(-0.3678)$ followed by days to last harvest ($0.3049)$ and fruit setting percent $(-0.2056)$ at genotypic level. Ascorbic acid content was recorded positive direct effect (0.2737) on fruit yield per plant. It had exerted highest positive indirect effect on fruit yield per plant through plant height (0.0883), fruit diameter (0.0767) and flavonoid content (0.0685).

The traits like number of branches per plant, plant spread (N-S), days to $50 \%$ flowering, number of fruit per plant, fruit pedicel length, ascorbic acid exhibited positive direct effects on fruit yield per plant and these traits recorded significant, positive correlation with fruit yield per plant. This suggested that direct selection based on these characters would be effective for an increase in yield, similar results were also reported in brinjal by Shende et al., (2014), Ravali et al., (2017) and Yadav et al., (2017).

Plant height, plant spread (EW), fruit setting percent, days to last harvest, fruit length, fruit diameter, total phenolic content and flavonoid content showed positive indirect effects on fruit yield per plant. This suggested that indirect selection based on these character will be effective in yield improvement. Similar results were reported in brinjal by Prabhu et al., (2008), Lakshmi et al., (2014) and Tripathy et al., (2018). Out of all characters studied, path analysis revealed that the traits like number of fruits per plant, number of branches per plant, plant spread $(\mathrm{N}-\mathrm{S})$, days to $50 \%$ flowering and ascorbic acid content was directly contributing to yield.

From the present investigation it is concluded that correlation coefficient and path coefficient for fruit yield per plant and its attributes in brinjal genotypes indicated the 
presence of ample variability for most of the traits. Correlation studies revealed that traits like plant height, number of branches per plant, plant spread, number of fruit per plant, days to $50 \%$ flowering, fruit length and diameter of fruit had significant association with fruit yield per plant. The result of path coefficient analysis indicated that the traits like number of fruits per plant, number of branches per plant, plant spread $(\mathrm{N}-\mathrm{S})$ and days to $50 \%$ flowering should be considered essential for developing high yielding genotypes as they had high positive direct effects on fruit yield per plant.

\section{Acknowledgement}

I would like to thank College of Agriculture, Parbhani and Horticulture Research Scheme (Vegetable) for providing all the materials and facilities throughout the course of this research work. I would also like to express my sincere gratitude to Dr. D.P. Waskar, Dr. V.S. Khandare Dr. S.P. Mehtre and Dr.D. G. More for their whole hearted support and guidance throughout the research work.

\section{References}

Anonymous (2019). Indian Horticulture Database. http:www.nhb.gov.in.

Dewey, D. R and K. N. Lu. (1959). A correlation and path coefficient analysis of components of crested wheat grass seed production. Agronomy Journal, 51: 515-518.

Gupta, R. A., C. N. Ram, Satish Kr. Chakravati, Chandra Deo, M. K. Vishwakarma, D. K. Gautam and Pushpendra Kumar. (2017). Studies on correlation and path coefficient analyses in brinjal (Solanum melongena L.). Int.J.Curr.Microbiol. App.Sci., 6(7): 4543-4548.

Johnson, H. W., H. F. Robinson and R. E Comstock. (1955). Genotypic and
Phenotypic correlation in soybean and their implications Selection. Agron.J., 47:477-485.

Kumar, R., K Anjali, A. K. Singh, S. Maurya. (2014). Screening of bacterial wilt resistant accessions of brinjal for Jharkhand region of India. The Ecoscan, 8(1-2): 67-70.

Lakshmi, R. R., S. S. Vijaya Padma, L. Naram Naidu and K. Umajyothi. (2014). Correlation and path analysis studies of yield and yield Components in brinjal. Plant Archives, 14(1): 583591.

Lenka D and B. Mishra. (1973). Path coefficient analysis of yield in rice varieties. Indian Journal of Agricultural Sciences. 43: 376-379.

Madhavi, N., J. Om Prasad, P. Bijalwan and V. Sekhar. (2016). Association analysis of yield and yield parameters in Brinjal (Solanum melongena L.). Plant Archives, 16(1): 426-430.

Pandey, P. K., G. C. Yadav and V. Kumar. (2016). Correlation and path coefficient analysis among different characters in genotypes of brinjal (Solanum melongena L.). Indian Journal of Ecology, 43(1): 370-372.

Patel, K., N. B. Patel, A. I. Patel, Hetal Rathod and Dharmishta Patel. (2015). Study of variability, correlation and path analysis in brinjal (Solanum melongena L.). The bioscan, 10(4): 2037-2042.

Prabhu, M., S. Natarajan and D. Veeraragavathatham. (2008). Correlation and path coefficient analysis in eggplant (Solanum melongena L.). Indian J. Agric. Res., 42 (3): $232-234$.

Ravali, B., P. Saidaiah, K. Ravinder Reddy, N. Shivraj and A. Geetha. (2017). Study on character association and path analysis in brinjal (Solanum melongena L.). Journal of 
Pharmacognosy and Phytochemistry, 6(6): 393-397.

Reshmika P. K. (2015). Genetic variability, divergence and correlation Studies in brinjal. International Journal of Agricultural Science and Research (IJASR) 5(6): 103-110.

Saleem, M.Y., I. Qumer and M. Apgar. (2013).Genetic variability, heritability, character association and path analysis in $\mathrm{f} 1$ hybrids of tomato. Pak. J. Agri. Sci., 50(4): 649-653.

Samadia, D. K. (2007). Genetic variability and correlation studies in brinjal. Indian journal of arid horticulture, 2(1): 18-21.

Sawadogo, B. P., Bationo-Kando, N. Sawadogo, Z. Kiebre, M. Kiebre, K. R. Nanema, R. E. Traore, M. Sawadogo And J. D. Zongo. (2016). Variation, correlation and heritability of interest characters for selection of African eggplant. African Crop Science Journal, 24(2): 213 - 221.

Shende, R. A., S. S. Desai, and V. V. Dalvi, (2014). Character association and path analysis in brinjal (Solanum melongena L.). Internat. J. agric. Sci., 10 (2): 631-633.

Shinde, K. G., S. D. Warade and J. H. Kadam. (2009). Correlation studies in brinjal (Solanum melongena L.). International Journal of Agricultural Sciences, 5(2): 507-509.

Sivakumar, V., K. Uma Jyothi, C. Venkataramana, M. Paratparao, R. Rajyalakshmi and K. Umakrishna. (2016).Character association and path co-efficient analysis studies on yield and attributing characters in brinjal (Solanum melongena L.). Electronic Journal of Plant Breeding, 7(3): 692696.

Sujin G. S., P. Karuppaiah and K. Saravanan. (2017). Genetic variability and correlation studies in brinjal(Solanum melongena L.). Indian J. Agric. Res., 51(2): 112-119.

Tembhurne, B. V., Revanappa and P. H. Kuchanur. (2008). Varietal performance, genetic variability and correlation studies in chilli (Capsicum annuumL.) Karnataka J. Agric. Sci., 21(4): 541-543.

Tripathy, B., D. Sharma, J. Singh and S. K. Nair. (2018). Correlation and path analysis studies of yield and yield components in brinjal (Solanum melongena L.).Int. J. Pure App. Biosci., 6(1): 1266-1270.

Umar Musa Tanko Momoh, Jimoh Yusuf. (2015). Correlation Studies on the Yield and Yield Characters of Eggplant (Solanum melongena L.) in Anyigba, Kogi State Nigeria. Developing Country Studies, 5(19): 2224-2225.

Yadav, N., S. K. Dhankhar, A. V. chandanshive and V. kumar. (2017). Character Association and Path Coefficients Analysis for Various Yields Attributes of Brinjal (Solanum melongena L.). International Journal of Agriculture Sciences, 9(7): 38363839 .

\section{How to cite this article:}

Gurve, V. R., D. P. Waskar, V. S. Khandare, S. P. Mehtre and More, D. G. 2020. Studies on Correlation and Path Analysis for Yield and its Contributing Traits in Brinjal (Solanum melongena L.). Int.J.Curr.Microbiol.App.Sci. 9(10): 179-188.

doi: https://doi.org/10.20546/ijcmas.2020.910.023 